\title{
Zebrafish (Danio rerio) behavioral laterality predicts increased short-term avoidance memory but not stress-reactivity responses
}

\author{
Barbara D. Fontana ${ }^{1}$ (C) $\cdot$ Madeleine Cleal $^{1}$ - James M. Clay ${ }^{3} \cdot$ Matthew O. Parker $^{1,2,3}$ (D)
}

Received: 11 February 2019 / Revised: 9 July 2019 / Accepted: 15 July 2019 / Published online: 24 July 2019

(c) The Author(s) 2019

\begin{abstract}
Once considered a uniquely human attribute, behavioral laterality has proven to be ubiquitous among non-human animals, and is associated with several neurophenotypes in rodents and fishes. Zebrafish (Danio rerio) is a versatile vertebrate model system widely used in translational neuropsychiatric research owing to their highly conserved genetic homology, wellcharacterized physiological responses, and extensive behavioral repertoire. Although spontaneous left- and right-biased responses, and associated behavioral domains (e.g., stress reactivity, aggression, and learning), have previously been observed in other teleost species, no information relating to whether spontaneous motor left-right-bias responses of zebrafish predicts other behavioral domains has been described. Thus, we aimed to investigate the existence and incidence of natural left-right bias in adult zebrafish, exploiting an unconditioned continuous free movement pattern (FMP) Y-maze task, and to explore the relationship of biasedness on performance within different behavioral domains. This included learning about threat cues in a Pavlovian fear conditioning test, and locomotion and anxiety-related behavior in the novel tank diving test. Although laterality did not change locomotion or anxiety-related behaviors, we found that biased animals displayed a different search strategy in the Y-maze, making them easily discernable from their unbiased counterparts, and increased learning associated to fear cues. In conclusion, we showed, for the first time, that zebrafish exhibit a natural manifestation of motor behavioral lateralization which can influence aversive learning responses.
\end{abstract}

Keywords Anxiety $\cdot$ Behavioral asymmetry $\cdot$ Free movement pattern Y-maze $\cdot$ Pavlovian fear conditioning $\cdot$ Laterality bias

\section{Introduction}

Lateralization of brain and behavior is the apparent predisposition towards side bias, which often manifests in terms of motor output, such as handedness. In humans, functional laterality of different brain regions is important in both language and cognition (Hull and Vaid 2007; Vikingstad et al.

Barbara D. Fontana

barbara.fontana@port.ac.uk

$\triangle$ Matthew O. Parker

matthew.parker@port.ac.uk

1 Brain and Behaviour Laboratory, School of Pharmacy and Biomedical Sciences, University of Portsmouth, White Swan Road, Portsmouth PO1 2DT, UK

2 The International Zebrafish Neuroscience Research Consortium (ZNRC), 309 Palmer Court, Slidell, LA 70458, USA

3 Department of Psychology, University of Portsmouth, King Henry I Street, Portsmouth PO1 2DY, UK
2000). Motor functions may also be under the control of lateralized mechanisms which may manifest as preference for one side over the other (e.g., handedness, footedness and eyedness) (Brown and Taylor 1988). Laterality is an evolutionarily conserved characteristic which is observed at the populational level and variance in laterality is associated with cognitive and neuropsychiatric disorders including anxiety and major depressive disorder (Koster et al. 2010; Lichtenstein-Vidne et al. 2017).

Lateralization is not a purely human characteristic. Most vertebrate species, including monkeys (Fagot and Vauclair 1991; Hopkins 1994; McGrew and Marchant 1997), rodents (Robison 1981; Rodriguez and Afonso 1993; Rodriguez et al. 1992), birds (Bhagavatula et al. 2014; Franklin and Adams 2010; Gunturkun et al. 1998), fishes (Bibost and Brown 2014; Bisazza and de Santi 2003; Dadda et al. 2010a, b), and some invertebrate species (Anfora et al. 2010; Frasnelli et al. 2012) express brain functional asymmetries. In rodents, for example, several behavioral tasks have been used to assess behavioral asymmetries such as 
turning rotometers, handedness, choice behavior, T-maze, and Y-maze (Corballis 1986; Pisa and Szechtman 1986; Zimmerberg and Glick 1974). Variability in lateralization exerts a number of fitness benefits at the individual level. For example, lateralization has been associated with maximization of brain processes, enabling individuals to process two tasks simultaneously (Rogers 2000, 2002). Studies have suggested that laterality evolved at the population level to maintain coordination among social groups. Commonality in the direction of lateralization at the group level is as a benefit to group cohesion, providing a potential mechanism, where individuals interact in predictable ways within the group (Rogers 2000).

Taxonomic and evolutionary aspects of brain laterality have been described in fish species, primarily focusing on CNS asymmetries, sensory organs and somatic lateralization, as well as the adaptive role of laterality in nature (de Perera and Braithwaite 2005; Lychakov 2013; Nepomnyashchikh and Izvekov 2006; Vallortigara and Rogers 2005). Behavioral asymmetries have been related to high escape performance (Dadda et al. 2010b), social responses (Reddon and Balshine 2010), and even accelerated learning responses (Andrade et al. 2001), in both fishes and mammals.

Zebrafish (Danio rerio) is a versatile vertebrate model system that has been widely used in translational neuropsychiatric research (Fontana et al. 2018; Stewart et al. 2015), and as a model to understand evolutionary aspects of animal cognition (Oliveira 2013; Reale et al. 2007). The last decade has seen an increase in the use of zebrafish to study the mechanisms underlying lateralization (Andersson et al. 2015; Ariyomo and Watt 2013; Barth et al. 2005; Dadda et al. 2010a; Sovrano and Andrew 2006). Larval zebrafish, for example, predominantly use the left-eye when interacting with their own reflection (Sovrano and Andrew 2006). In addition, zebrafish initially use the right hemifield predominantly when interacting with novel objects, but as the object becomes familiar, they switch to the left hemifield (Miklósi et al. 1997). Despite a growing understanding of developmental biology of lateralization in zebrafish (e.g., visual lateralization in learning and memory) (Andersson et al. 2015), very little is known about how spontaneously occurring motor asymmetry impacts on adult zebrafish behavior. Therefore, the principal aims of the present study were: (1) to investigate the existence and incidence of spontaneously occurring motor left-right bias of adult zebrafish in an unconditioned continuous free movement pattern (FMP) Y-maze task and (2) to explore how spontaneously occurring motor left-right bias relates to performance on different behavioral domains, including learning about threat cues in the fear conditioning test, and locomotion and anxiety-related behavior in the novel tank test.

\section{Materials and methods}

\section{Animals}

Adult zebrafish (AB wild type; 50:50 male:female ratio at 3 months of age) were bred in-house and reared in the standard laboratory conditions on a re-circulating system (Aquaneering, USA). Animals were maintained on a 14/10-h light/dark cycle (lights on at 9:00 a.m.), pH 8.4, at $\sim 28.5{ }^{\circ} \mathrm{C}\left( \pm 1{ }^{\circ} \mathrm{C}\right)$ in groups of 20 animals per $2.8 \mathrm{~L}$. Fish were fed three times/day with a mixture of live brine shrimp and flake food, except at weekends when they were fed once a day. Animals were tested in the FMP Y-maze apparatus and then pair-housed for $24 \mathrm{~h}$ prior to analysis of shock avoidance or tank diving, to reduce stress from multiple handling in a single day (see Fig. 1). During the pair-housing period, animals had shared water system and visual contact through transparent partition, the pair-housing system is used to separate animals for further identification and reduce the stress induced by social isolation (Parker et al. 2012). After behavioral tests, all animals were euthanized using 2-phenoxyethanol from Aqua-Sed (Aqua-Sed $^{\mathrm{TM}}$, Vetark, Winchester, UK). All experiments were carried out following approval from the University of Portsmouth Animal Welfare and Ethical Review Board, and under license from the UK Home Office [Animals (Scientific Procedures) Act, 1986] [PPL: P9D87106F].

\section{Free movement pattern Y-maze task}

Y-maze spontaneous alternation tasks have previously been used to assess left- and right-biased responses (Barnard et al. 2016; Castellano et al. 1987; Frasnelli 2013; Rodriguez et al. 1992). Here, we used a novel version of the Y-maze developed in our lab. In the FMP Y-maze task, fishes behavior patterns were recorded for $1 \mathrm{~h}$. Choices at each fork in the maze were binary and could either be 'left' or 'right', and thus represent a series of non-discrete choice "trials" within the continuous search task. Choices were quantified for each fish in terms of continuously overlapping series of four choices (tetragrams; 111l, 11lr, 1lrl, [etc.]..., rrrr) (Cleal and Parker 2018; Gross et al. 2011). Analysis was normalized by calculating frequency of each tetragram as a proportion of the total number of turns. For assessing fishes' behavioral lateralization, the number of turns to the right or left was calculated. To calculate index of bias, choices (in 10-min time bins) were entered into the following formula: Bias percentage $=\frac{\text { number of turns } L \text { or } R \times 100}{\text { total number of turns }}$. Following this calculation, the mean and coefficient of variation (CV) for right and left bias were calculated across 


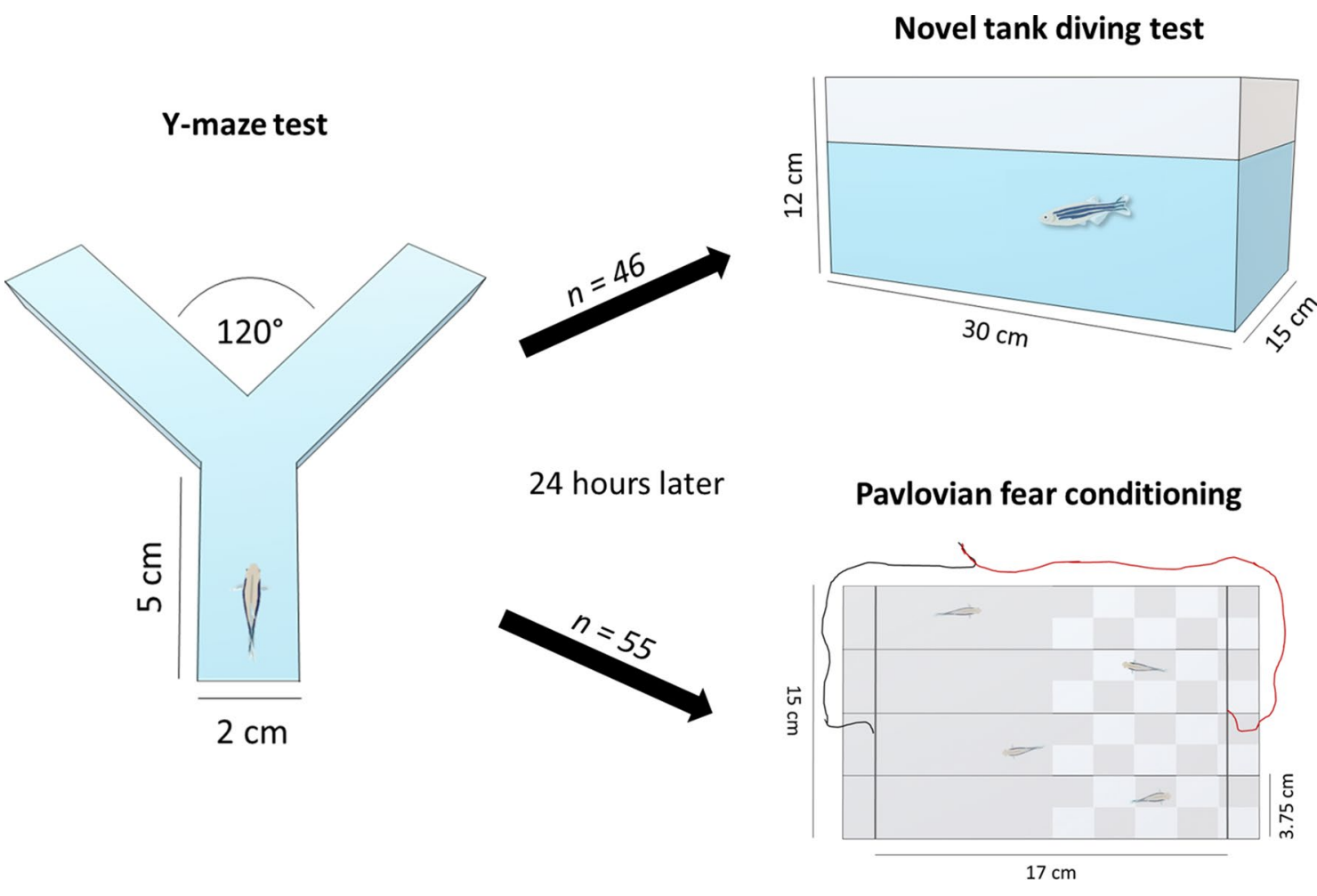

Fig. 1 Schematic representation of the experimental design and the behavioral tasks

the 10 -min time bins to assess the consistency of data across the entire 1 -h test period.

One-hundred and three adult zebrafish were used for assessing FMP Y-maze performance and right-left bias. Required sample size was calculated a priori following pilot tests, in which we examined the relative frequency of biased animals (showing $>60 \%$ side bias) to unbiased [effect size $(d)=0.3$, power $=0.8$, alpha $=0.05]$. Each fish was used only once, and we carried out three complete independent batches on different days. Behavioral tests were performed between 10 a.m. and 4 p.m. using the Zantiks [AD] fully automated behavioral testing environment (Zantiks Ltd., Cambridge, UK; Brock et al. 2017). The Zantiks AD system was fully controlled via a web-enabled device during behavioral training. The test tank consisted of two identical white plastic Y-mazes, each with three identical arms $(5 \mathrm{~cm}$ length $\times 2 \mathrm{~cm}$ width; arms at a $120^{\circ}$ angle from each other) and a transparent base (Fig. 1). The tank in which the maze was located was filled with $3 \mathrm{~L}$ of aquarium water. Ambient light (44 1x) allowed some visibility in the maze, but no explicit intra-maze cues were added, to ensure that each fishes' perceived location remained ambiguous, regardless of its relative position in the maze. Behavioral lateralization was considered when the fish carried out $>60 \%$ of right or left turns during the 1-h test. To assess zebrafish search strategies, we used the number of alternations ( $r l r l+l r l r)$ and repetitions ( $\mathrm{rrr}+111 \mathrm{l})$ as proportion of total number of turns which are highly expressed through $1 \mathrm{~h}$ and are commonly linked to animals' exploratory activity (Cleal and Parker 2018; Gross et al. 2011). Relative alternations have been suggested to be a measure of working memory, with a relative reduction the number of alternations using the tetragram methodology having been observed following developmental alcohol exposure (Cleal and Parker 2018), while relative repetitions are suggested to be a measure of response perseveration (Gross et al. 2011).

\section{Pavlovian fear conditioning}

The Pavlovian inhibitory avoidance paradigm is a valid method widely used to explore mechanisms underlying fear avoidance learning responses in zebrafish (Amorim et al. 2017; Manuel et al. 2014, 2015; Ng et al. 2012). Twentyfour hours after the completion of the FMP Y-maze task, fish $(n=55)$ were tested on a Pavlovian fear conditioning procedure for $1 \mathrm{~h}$. The fear conditioning response was based on previous work (Cleal and Parker 2018; Valente et al. 2012). Fish were individually placed in one of the four lanes of a tank $(25 \mathrm{~cm}$ length $\times 15 \mathrm{~cm}, 1 \mathrm{~L}$ of water in each tank) of the Zantiks (Zantiks Ltd., Cambridge, UK) AD system (Brock et al. 2017) (Fig. 1). Briefly, fishes were habituated for $30 \mathrm{~min}$ in the test environment, during which time, half of the arena had a check pattern on the base and the other half had a grey color on the base (alternating between sides 
of the tank every $5 \mathrm{~min}$ ). Images were displayed on a screen in the base of the AD unit, on which the test tank sits. Following 30-min habituation, 10-min baseline data were collected to assess preference for the stimuli. Following the baseline was the conditioning phase, which consisted of a conditioned stimulus (CS+ ; full screen of "check" or "grey" [half CS+ in "check" and CS+ in "grey"]) presented for $1.5 \mathrm{~s}$ and followed by a brief mild shock ( $9 \mathrm{~V} \mathrm{DC}, 80 \mathrm{~ms}$; unconditioned stimulus (US). After this, an 8.5-s inter-trial interval (ITI) of the non-CS (CS-) exemplar was presented at the bottom of the tank. The CS+/US was presented nine times. Finally, avoidance of CS+ was assessed by presenting $\mathrm{CS}+$ and CS - simultaneously for $1 \mathrm{~min}$ at either end of the tank (switching positions after $30 \mathrm{~s}$ ) and calculating the time spent in the vicinity of $\mathrm{CS}+$, and comparing this to baseline preference.

\section{Novel tank diving and thigmotaxis responses}

The novel tank test measures locomotor and anxiety-like phenotypes in zebrafish, and is highly sensitive both to anxiolytic and anxiogenic drugs (Egan et al. 2009; Kalueff et al. 2013; Levin et al. 2007; Maximino et al. 2010; Mezzomo et al. 2016; Wong et al. 2010). Twenty-four hours after the FMP Y-maze test, animals $(n=46)$ were placed individually in a novel tank $(30 \mathrm{~cm}$ length $\times 15 \mathrm{~cm}$ height $\times 12 \mathrm{~cm}$ width) containing $4 \mathrm{~L}$ of aquarium water. Behavioral activity was recorded using two webcams (front and top view, see Fig. 1b) for 5 min to analyze thigmotaxis and diving response (Egan et al. 2009; Parker et al. 2012; Rosemberg et al. 2012). Behaviors were measured using an automated video-tracking software (EthoVision, Noldus Information Technology Inc., Leesburg, VA-USA) at a rate of 60 frames/s. Thigmotaxis (time spent in proximity to the edge/sides) was analyzed through behavioral tracking obtained by the top view camera. Meanwhile, for a detailed evaluation of vertical activity, the tank was separated in three virtual areas (bottom, middle, and top) and the following endpoints were measured: total distance traveled, time spent in each third of the tank, and immobility.

\section{Randomization and blinding}

All behavioral testing was carried out in a fully randomized order, choosing fish at random from one of the ten housing tanks for testing. Fish were screened for left-right bias in the FMP Y-maze first, but analysis was not carried out prior to subsequent behavioral testing to avoid bias. Subsequent to the FMP Y-maze screening, fish were pair-housed and issued a subject ID, allowing all testing to be carried out in a fully blinded manner. Once all data were collected and screened for extreme outliers (e.g., fish freezing and returning values of ' 0 ' for behavioral parameters indicating non-engagement), the bias was revealed, and data analyzed in full. Two animals were excluded in the FMP Y-maze task and two in the novel tank due to poor engagement with the task (freezing and displaying no measurable behavioral patterns).

\section{Data processing and statistical analysis}

Raw data from the FMP Y-maze task was in the form of arm entries. To analyze the data according to left and right turns in 10-min time bins, raw data were processed using a custom-written R (www.r-project.org) script (available from: https://github.com/thejamesclay/ZANTIKS_YMaze_Analy sis_Script). Subsequently, data were analyzed in IBM SPSS ${ }^{\circledR}$ Statistics and the results were expressed as mean \pm standard error of the mean (S.E.M), to assess whether there were any effects of 'bias' (>60\% preference for left/right) on total turns, alternations $(\mathrm{lrlr}+\mathrm{rlrl})$ or repetitions $(\mathrm{rrrr}+\mathrm{llll})$. Alternations and repetitions were analyzed using generalized linear models (Poisson distribution and log link), with laterality (three levels-left bias, right bias, and non-bias) and time (six levels-10-min time bins across $1 \mathrm{~h}$ ) as the fixed factors, and ID as a random effect (to account for non-independence of replicates). To analyze novel tank responses, we used either one- or two-way ANOVAs, with bias and time spent in different tank zones fixed factors (novel tank) or bias only as a fixed factor for thigmotaxis, immobility, and distance traveled. In addition, left-right-bias effects on the shock-avoidance test were assessed using two-way repeated measures ANOVA with 'bias' (left vs right vs neutral) and conditioning (pre vs. post) as factors, and preference for conditioned stimulus as the dependent variable. Newman-Keuls test was used as post hoc analysis, and results were considered significant when $p \leq 0.05$.

\section{Results}

\section{Left-right-bias profile in the FMP Y-maze test}

Zebrafish showed behavioral lateralization in the FMP Y-maze (right biased $27.18 \%$, left-biased $27.18 \%$ and nonbiased $45.63 \%)$. First, we confirmed that behavioral laterality was consistent across $1 \mathrm{~h}$ (analyzed in 10-min time bins), by calculating the coefficient of variation (CV) for the left and right-turn preferences for the non-biased (left CV 19.28 \pm 2.52 and right CV 21.05 \pm 2.95 ), left-biased (left CV $30.40 \pm 3.85$ and right CV $21.7 \pm 3.61$ ), and right biased (left CV 27.23 \pm 5.52 and right CV 25.95 \pm 2.31 ) groups. Figure 2 displays the FMP Y-maze data. A significant bias effect was observed for number of turns $\left[F_{(2,601)}=13.115\right.$; $p<0.0001]$, repetitions $\left[F_{(2,601)}=39.696 ; p<0.0001\right]$, and alternations $\left[F_{(2,601)}=45.437 ; p<0.0001\right]$. A time effect (data not shown) was also observed for number of turns 

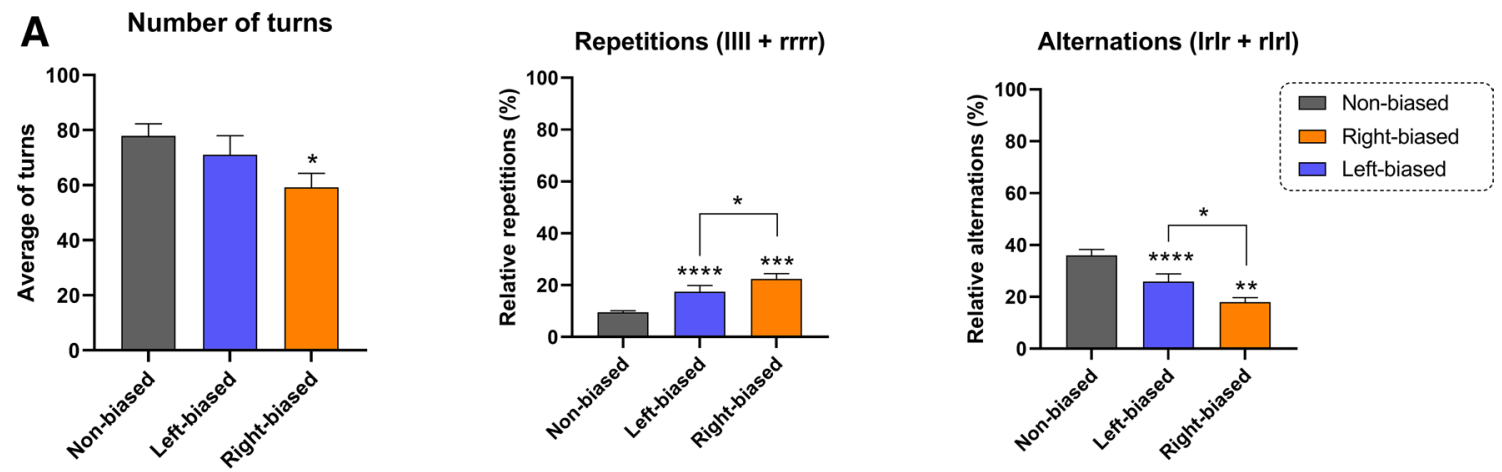

B

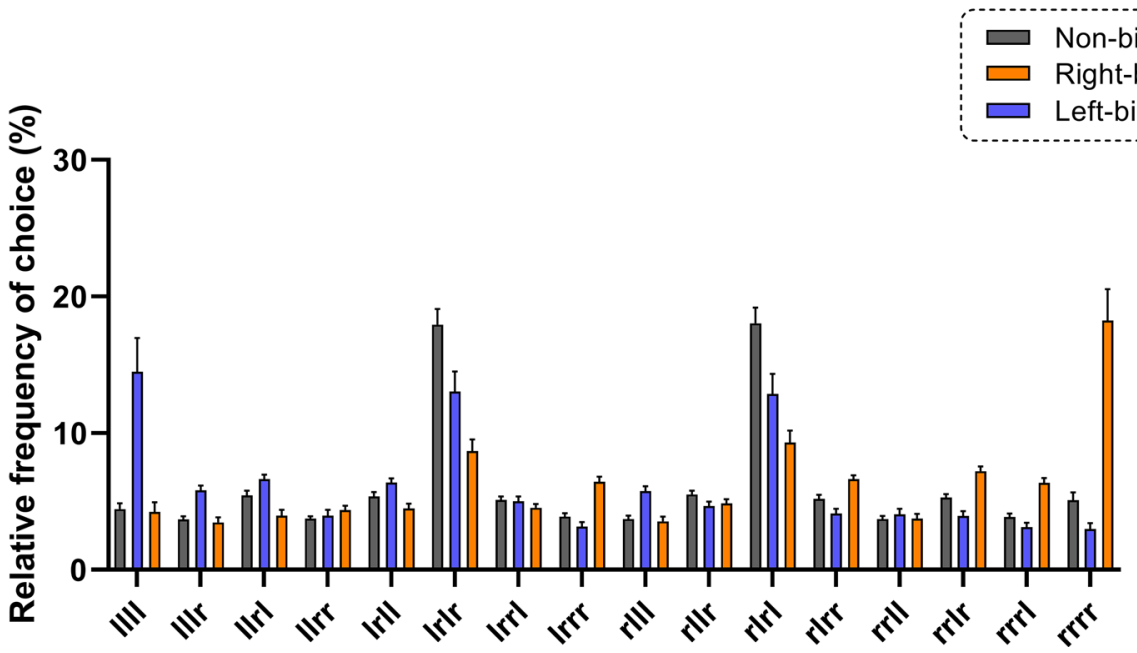

Fig. 2 Effects of left- and right bias in zebrafish on the Y-maze test. a Laterality affects total number of turns, repetitions and alternation of adult zebrafish. b Y-maze tetragrams showing the behavioral phenotype of biased and non-biased animals considering the relative frequency of choice (tetragram frequency of choice $\times 100 /$ total number of turns). Data were represented as mean \pm S.E.M. and ana-

$\left[F_{(5,601)}=9.769 ; p<0.0001\right]$, repetitions $\left[F_{(5,601)}=3.242\right.$; $p=0.007]$, and alternations $\left[F_{(5,601)}=3.801 ; p=0.002\right]$. In addition, a significant interaction effect (bias $\times$ time) was observed for repetitions $\left[F_{(10,601)}=2.504 ; p=0.006\right]$ and alternations $\left[F_{(10,601)}=2.390 ; p=0.009\right]$. Bias, to the left or the right, significantly increased the number of repetitions ( $p<0.0001$ for right bias and $p<0.001$ for left bias) and decreased the percentage of alternations $(p<0.0001$ for right bias and $p<0.005$ for left bias) compared to nonbiased animals. In addition, right-biased fish decreased the number of turns $(p<0.05)$ compared to non-biased fish. Differences were not just observed between biased vs. nonbiased groups, but also between bias groups, with rightbiased fish displaying a significant increase in repetitions $(p<0.05)$ and decrease in alternations $(p<0.05)$ compared to left-biased fish (Fig. 2a). The behavioral profile of biased and non-biased fish is displayed in terms of tetragrams (Fig. 2b), where the relatively high number of 1111 and rrrr lyzed by linear mixed effects, followed by Tukey's multiple comparison test. Asterisks indicates statistical differences compared to non-biased group or between biased groups $\left({ }^{*} p<0.05,{ }^{* *} p<0.01\right.$, $* * * p<0.001$ and $* * * * p<0.0001, n=47$ non-biased, $n=28$ leftbiased and $n=28$ right-biased group)

configurations can be observed for left- and right-biased animals, respectively.

\section{Short-term avoidance memory and novelty response of biased animals}

Although no interaction effect bias vs. shock $\left[F_{(2,98)}=1.259\right.$; $p=0.312]$ was observed for the Pavlovian responses, a significant effect for bias $\left[F_{(2,98)}=3.128 ; p=0.035\right]$ and conditioning (probe vs. baseline) $\left[F_{(1,98)}=79.47 ; p<0001\right]$ effect was revealed. ANOVA analyses are often underpowered and, therefore, unable to detect the significance of interaction terms (Wahlsten 1990). Therefore, post hoc analysis was performed to specifically analyze the effects between groups. In general, all biased ( $p<0.0001$ for right- and left bias) and non-biased $(p<0.0001)$ animals had a decreased time spent in the preference for conditioned stimulus (probe vs. baseline). However, both left- and right-biased animals 


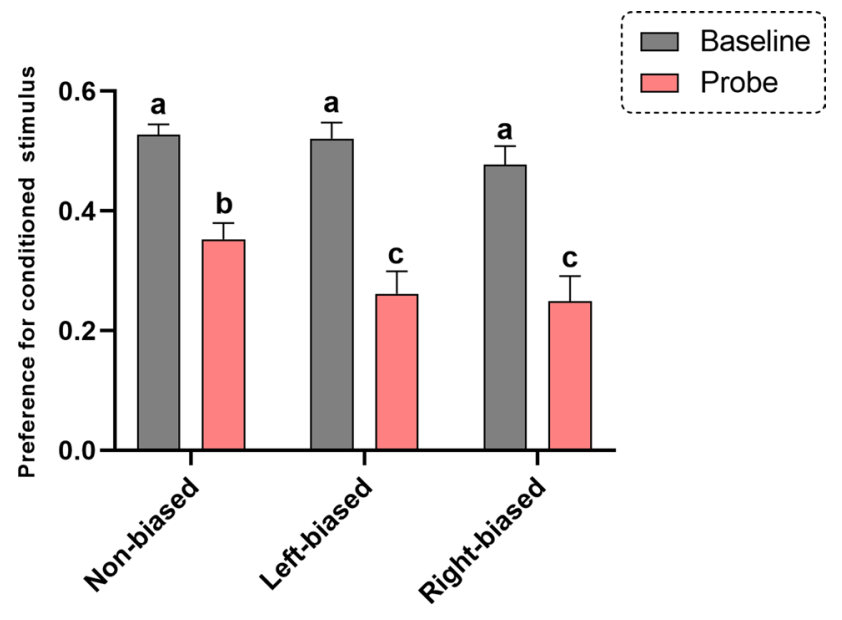

Fig. 3 Left- and right bias are related to fear avoidance learning responses in adult zebrafish. Data were represented as mean \pm S.E.M. and analyzed by two-way RM ANOVA, followed by Tukey's multiple comparison test. Different letters indicate significant differences between groups $(p<0.05 ; n=25$ non-biased, $n=17$ left-biased and $n=13$ right-biased group)

$(p<0.05)$ spent significantly less time in the conditioned area during the probe trial compared to non-biased group (Fig. 3), despite no significant differences in their baseline preferences, suggesting a stronger response to the aversive stimulus. No significant effect was observed for bias in all novel tank diving test-related parameters, including distance traveled $\left[F_{(2,43)}=0.683 ; p=0.510\right]$, immobility $\left[F_{(2,43)}=2.348 ; p=0.107\right]$, time in tank zones time $\left[F_{(2,129)}=0.084 ; p=0.918\right]$ (Fig. 4), and thigmotaxis $\left[F_{(2,43)}=1.289 ; p=0.286\right]$ (Fig. 5).

\section{Discussion}

In this study, we evaluated spontaneously occurring motor left-right bias in adult zebrafish using the continuous unconditioned FMP Y-maze task, and evaluated the predictive validity of spontaneous behavioral laterality on both unconditioned and conditioned measures of fear and anxiety. We showed, for the first time, that the zebrafish presents spontaneous behavioral laterality in the FMP Y-maze test when analyzed using 16 overlapping tetragrams to evaluate exploration strategy, suggesting that the protocol may be useful for screening this species for behavioral asymmetry. Second, we found that behaviorally lateralized animals show decreased alternations and increased repetitions patterns in the FMP Y-maze compared to non-biased animals. This suggests that search strategies used by spontaneously biased individuals differ from that used by non-biased fish and is quite likely directly due to the lateralization. Third, we observed that behavioral asymmetry predicts increased aversive responses in a Pavlovian fear conditioning protocol, but did not predict measures of unconditioned anxiety (novel tank test, thigmotaxis). Collectively, these data suggest, contrary to theories that laterality bias in fish is related to increased stress-reactivity, that increased behavioral laterality may be related to increased cue reactivity, particularly in relation to aversive cues. This has connotations for translational models of human disorders of affective state, in which heightened attention to threat-related cues is observed (Lichtenstein-Vidne et al. 2017) and in which variations in brain and behavioral laterality are thought to be risk factors (Bruder et al. 2016).

Left-right asymmetries in behavioral protocols including the T-maze and Y-maze have been widely utilized in rodents (Andrade et al. 2001; Nakagawa et al. 2004; Rodriguez et al. 1992). Here, for the first time, we observed that approximately a quarter of zebrafish present substantial natural left- $(27.18 \%)$ or right- $(27.18 \%)$ locomotor lateralization, with the remaining $45.63 \%$ of animals showing stochastic patterns of left/right. These data are somewhat at odds from observed bias in other models, in which there is a high number of right-biased animals (52.8\%) and low numbers of left- $(22.2 \%)$ and non-biased responses (25\%) for rodents (Andrade et al. 2001). Using an open field detour test (Chivers et al. 2016) showed that wild-caught fish, yellowand-blueback fusiliers (Caesio teres) present a spontaneous increased bias for right turns $(65 \%)$ and decreased bias for left turns (24\%), with no left/right bias representing the least common phenotype (11\%). However, they also observed that the strength of the behavioral lateralization was increased depending on predation pressure and that right-biased fish, in general, displayed better escape performance as compared to left-biased and non-biased individuals (Chivers et al. 2016).

Studies focusing on the evolutionary aspects of lateralization suggest that behavioral asymmetries exist at population level, because individually asymmetrical organisms coordinate their behavior with other asymmetrical organisms (Frasnelli 2013). For example, right-biased animals showed increased escape responses (Chivers et al. 2016) and increased right-eye use when attacking the mirror image aggression (Bisazza and de Santi 2003). In invertebrates, laterality has also been consistent with the vertebrate findings, where eusocial ants show motor left bias when exploring unfamiliar nest sites (Hunt et al. 2014), and eusocial honeybees have olfactory asymmetries that predict learning and recall of memory (Frasnelli et al. 2010; Rigosi et al. 2011; Rogers and Vallortigara 2008).

We observed that behavioral laterality has an important role in FMP Y-maze performance, where left-right-biased animals presented an increase of repetition behavior and decrease of alternation, in particular in the left-biased animals. Previous studies (Cleal and Parker 2018; Gross et al. 

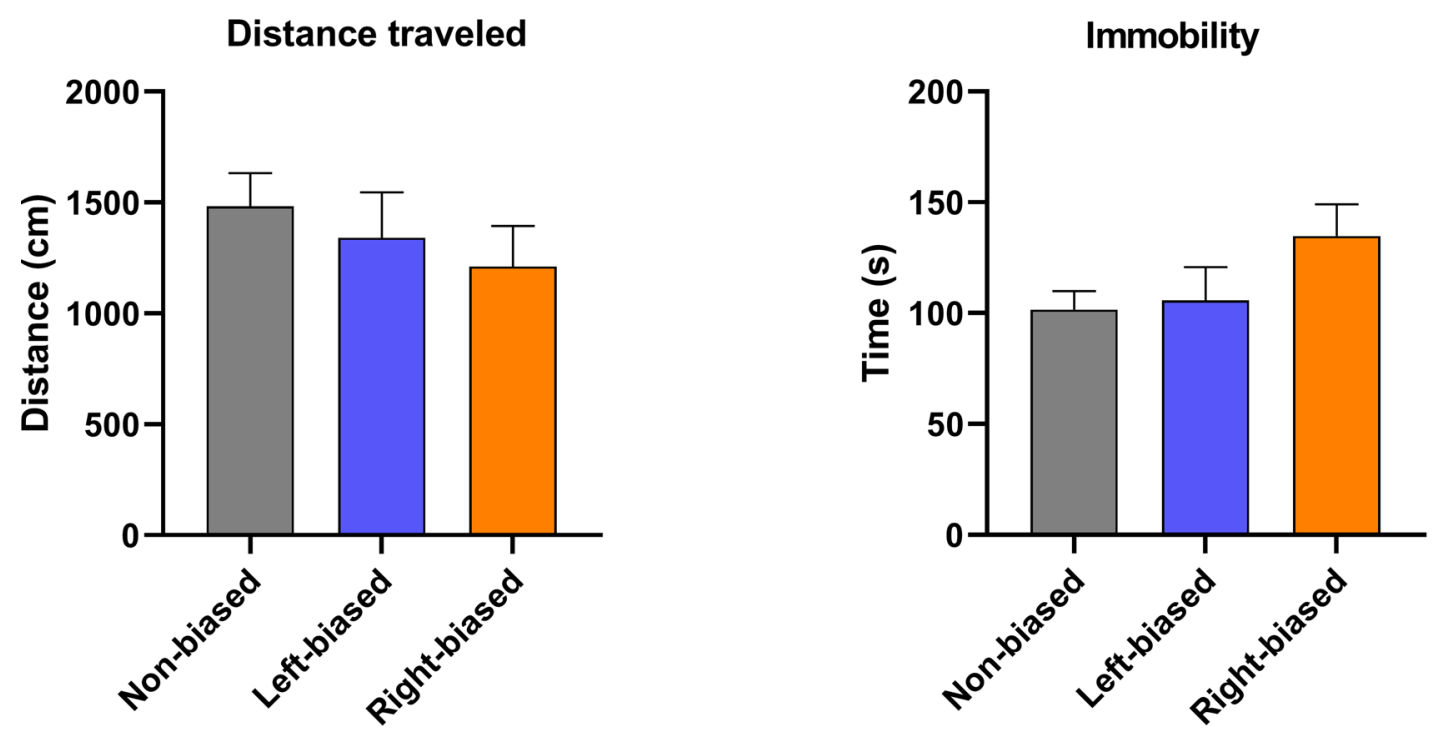

Time in tank zones

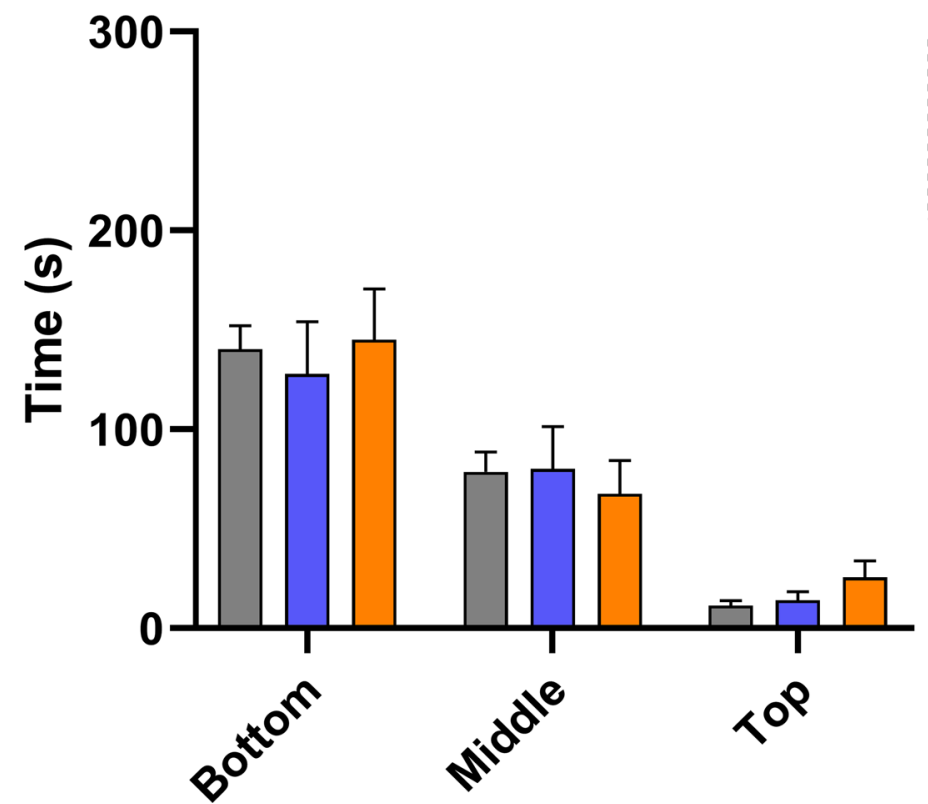

Fig. 4 Behavioral laterality is not related to locomotor or anxiety-related phenotypes in adult zebrafish. Data were represented as mean \pm S.E.M. and analyzed by one- or two-way ANOVA ( $n=22$ non-biased, $n=11$ left-biased and $n=15$ right-biased group)

2011) and continuing work in our laboratory, in conjunction with rodent $\mathrm{T}$ - and Y-maze data, suggest that typical spontaneous search strategy employed is to use a relatively high level of alternations. Alternations have been directly associated with functionally distinct search patterns, where the seeking for change and novelty may have a role in their exploratory profiles (Kool et al. 2010). Rodriguez et al. (1992) described decreases in pure alternations and increases in pure repetition behavior in lateralized animals, and demonstrated that both stress and over-training decrease the alternation/repetition ratio through the promotion of an increase of biased responses. Although the dominant strategy, alternations and, to a far lesser extent, repetitions, are punctuated with other more stochastic patterns, as indicated in Fig. 2b showing the relative frequency of each of the 16 tetragrams throughout the $1-\mathrm{h}$ trial. We have suggested before that the dominant search strategies used in the FMP Y-maze task are associated with motor working memory (alternations) or perseveration (repetitions) (Cleal and Parker 2018). This theory of what underlies the dominant strategies 


\section{Thigmotaxis}

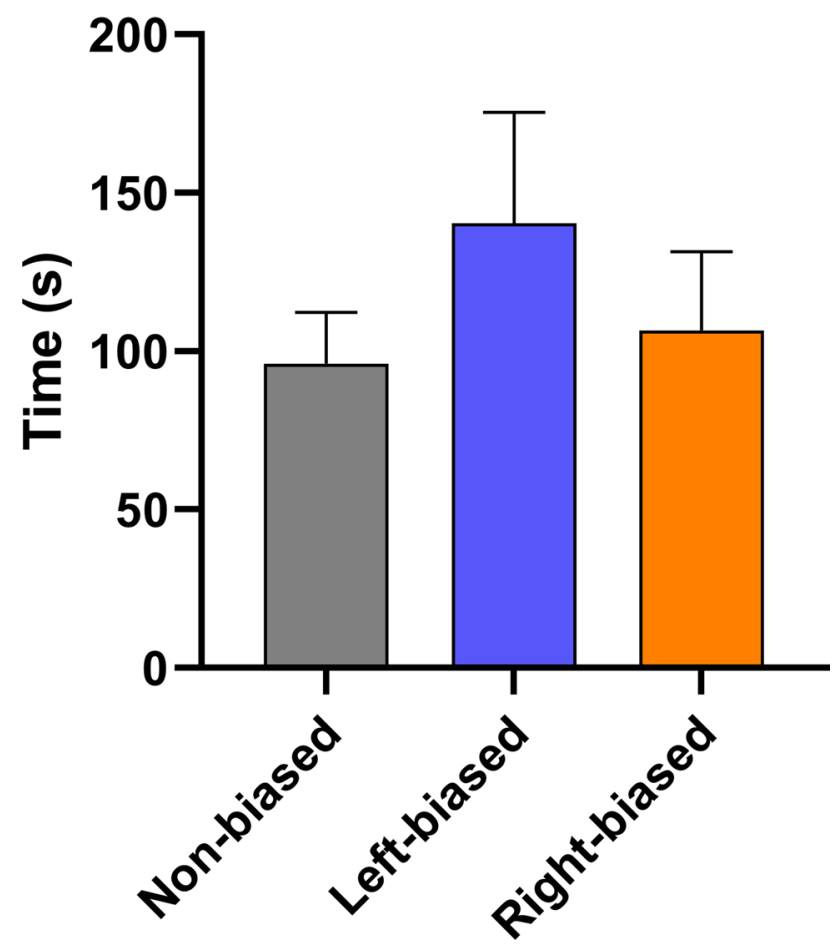

Fig. 5 Left- and right bias do not change thigmotaxis in adult zebrafish. Data were represented as mean \pm S.E.M. and analyzed by one-way ANOVA ( $n=20$ non-biased, $n=11$ left-biased and $n=15$ right-biased group)

would suggest that lateralized zebrafish are more strategically perseverative, as opposed to using working memory. However, further work would have to be carried out to determine the mechanisms underlying alternations and repetitions in FMP Y-maze performance. Here, we confirmed that both alternations and repetitions remain as a highly reliable behavioral pattern that is conserved across species, and that variations in FMP Y-maze may be a fruitful direction for more detailed analysis in the future (Ghafouri et al. 2016; Lewis et al. 2017; Pickering et al. 2015).

In agreement with the previous studies (Andrade et al. 2001), we showed that locomotor lateralization is associated with increased learning in a Pavlovian fear conditioning protocol. Studies using other fish species showed that lateralized animals have a better response in cognitive tasks such as spatial reorientation (Sovrano et al. 2005), and lefteye bias is related to faster learning in conditioning tasks (Bibost and Brown 2014). In general, the most predominant theory of how left-right bias affects learning and cognitive processing relates to a hypothesized increased stress-reactivity in lateralized animals (Carlson and Glick 1989; Neveu 1996; Westergaard et al. 2001). Interindividual differences in laterality have been shown to covary with, or predict, individual differences in stress-reactivity and susceptibility to stress-related pathology (Byrnes et al. 2016; Carlson and Glick 1989; Fride and Weinstock 1989; Ocklenburg et al. 2016). Here, we tested the hypothesis that left- and rightbiased animals would differ in measures of stress-reactivity and anxiety-like phenotypes (Blaser and Rosemberg 2012; Egan et al. 2009; Parker et al. 2012). We found no significant differences in lateralized animals in our measures of anxiety, suggesting that the observed differences in behavioral phenotypes observed in the FMP Y-maze and Pavlovian avoidance learning seems to not be related to stress-reactivity responses per se. Instead, our data may suggest that the lateralized fish are more reactive to stress-related cues. This would explain the increased performance in the Pavlovian fear conditioning, as well as the fact that there were no differences in measures of general anxiety. In addition, if the lateralized fish perceived the Y-maze as a more threatening or 'negative' environment, this may explain their change in search strategy: the role of stress in perseveration is well established (Ridley 1994).

There are several theories regarding the mechanisms underlying behavioral laterality in simple maze tasks. Diaz Palarea et al. (1987) were the first to report that left-rightbiased animals, as assessed via spatial asymmetry in a T-maze, had alterations in dopaminergic (DA) signaling. In addition, apomorphine (non-specific DA receptor agonist) and 6-hydroxydopamine lesions alters behavioral laterality of animals in the T-maze test (Castellano et al. 1987) and Y-maze (Nakagawa et al. 2004), confirming the involvement of DA system in behavioral asymmetry. DA receptors are strongly implicated in emotional learning and recall of emotionally relevant events in rats. For example, activation of D4-receptors in the medial pre-frontal cortex potentiates fear-associated memory formation, but has no impact on recall (Lauzon et al. 2009; Laviolette et al. 2005), whereas activation of D1-like receptors blocks recalls of previously learned fear-associated memories, but has no impact on learning (Lauzon et al. 2009), suggesting a double dissociation of function. Interestingly, the serotonergic (5-HT) system has also been shown to have an important role in mediating individual differences in anxiety-like responses and locomotor activity in zebrafish and exerts a minor modulatory role of the DA system (Tran et al. 2016). Both behavioral laterality and aversive memory is mostly associated with modulatory action of the DA system, but the 5-HT system has a major role modulating zebrafish responses to novelty. The precise mechanisms of how behavioral laterality modulates neuropsychiatric conditions are yet to be firmly established, and further studies are required to better understand the mechanisms in which behavioral laterality modulates aversive memory in zebrafish. Our data have shown that the FMP Y-maze may be a very useful tool for carrying out such research and should be exploited in the future. 


\section{Conclusion}

We showed for the first time that zebrafish exhibit spontaneously occurring motor lateralization which can influence aversive learning responses. We also found that biased animals show an altered exploratory search strategy in the FMP Y-maze, including higher repetitions and lower alternations. Coupled with a lack of observed differences between lateralized and non-lateralized animals in unconditioned tests of anxiety, our data suggest that lateralized zebrafish may show heightened reactivity to fear related cues. These results have important connotations for translational models of depression and anxiety, particularly in the light of well-established links between laterality and anxiety/depression in humans. Finally, because biased animals present different behavioral performances in the FMP Y-maze and Pavlovian fear conditioning protocols, leftand right- preference should be considered when working with zebrafish behavior, particularly to control variability in performance on more complex tasks.

Funding This study was financed in part by the Coordenação de Aperfeiçoamento de Pessoal de Nível Superior-Brazil (CAPES)—Finance Code 001 at the University of Portsmouth, UK. MOP receives funding from the Foundation for Liver Research and the British Academy. MC is supported by a Science Faculty Studentship from the University of Portsmouth.

\section{Compliance with ethical standards}

Conflict of interest The authors declare that no conflict of interest exists.

Ethical approval All experiments were carried out following scrutiny by the University of Portsmouth Animal Welfare and Ethical Review Board, and under license from the UK Home Office [Animals (Scientific Procedures) Act, 1986] [PPL: P9D87106F].

Open Access This article is distributed under the terms of the Creative Commons Attribution 4.0 International License (http://creativeco mmons.org/licenses/by/4.0/), which permits unrestricted use, distribution, and reproduction in any medium, provided you give appropriate credit to the original author(s) and the source, provide a link to the Creative Commons license, and indicate if changes were made.

\section{References}

Amorim RR, Silva PF, Luchiari AC (2017) Effects of alcohol on inhibitory avoidance learning in zebrafish (Danio rerio). Zebrafish 14:430-437. https://doi.org/10.1089/zeb.2017.1438

Andersson MA, Ek F, Olsson R (2015) Using visual lateralization to model learning and memory in zebrafish larvae. Sci Rep 5:8667. https://doi.org/10.1038/srep08667

Andrade C, Alwarshetty M, Sudha S, Suresh Chandra J (2001) Effect of innate direction bias on T-maze learning in rats: implications for research. J Neurosci Methods 110:31-35
Anfora G, Frasnelli E, Maccagnani B, Rogers LJ, Vallortigara G (2010) Behavioural and electrophysiological lateralisation in a social (Apis mellifera) but not a non-social (Osmia cornuta) species of bee. Behav Brain Res 206:236-239. https://doi.org/10.1016/j. bbr.2009.09.023

Ariyomo TO, Watt PJ (2013) Aggression and sex differences in lateralization in the zebrafish. Anim Behav 86:617-622

Barnard S, Matthews L, Messori S, Podaliri-Vulpiani M, Ferri N (2016) Laterality as an indicator of emotional stress in ewes and lambs during a separation test. Anim Cogn 19:207-214. https://doi. org/10.1007/s10071-015-0928-3

Barth KA, Miklosi A, Watkins J, Bianco IH, Wilson SW, Andrew RJ (2005) Fsi zebrafish show concordant reversal of laterality of viscera, neuroanatomy, and a subset of behavioral responses. Curr Biol CB 15:844-850. https://doi.org/10.1016/j.cub.2005.03.047

Bhagavatula PS, Claudianos C, Ibbotson MR, Srinivasan MV (2014) Behavioral lateralization and optimal route choice in flying budgerigars. PLoS Comput Biol 10:e1003473. https://doi.org/10.1371/ journal.pcbi.1003473

Bibost AL, Brown C (2014) Laterality influences cognitive performance in rainbowfish Melanotaenia duboulayi. Anim Cogn 17:1045-1051. https://doi.org/10.1007/s10071-014-0734-3

Bisazza A, de Santi A (2003) Lateralization of aggression in fish. Behav Brain Res 141:131-136

Blaser RE, Rosemberg DB (2012) Measures of anxiety in zebrafish (Danio rerio): dissociation of black/white preference and novel tank test. PloS One 7:e36931. https://doi.org/10.1371/journ al.pone. 0036931

Brock AJ, Goody SMG, Mead AN, Sudwarts A, Parker MO, Brennan $\mathrm{CH}$ (2017) Assessing the value of the zebrafish conditioned place preference model for predicting human abuse potential. J Pharmacol Exp Ther 363:66-79. https://doi.org/10.1124/jpet.117.242628

Brown ER, Taylor P (1988) Handedness, footedness, and eyedness. Percept Mot Skills 66(1):183-186. https://doi.org/10.2466/ pms.1988.66.1.183

Bruder GE et al (2016) Brain laterality, depression and anxiety disorders: new findings for emotional and verbal dichotic listening in individuals at risk for depression. Laterality 21:525-548. https:// doi.org/10.1080/1357650x.2015.1105247

Byrnes EE, Vila Pouca C, Brown C (2016) Laterality strength is linked to stress reactivity in Port Jackson sharks (Heterodontus portusjacksoni). Behav Brain Res 305:239-246. https://doi. org/10.1016/j.bbr.2016.02.033

Carlson JN, Glick SD (1989) Cerebral lateralization as a source of interindividual differences in behavior. Experientia 45:788-798

Castellano MA, Diaz-Palarea MD, Rodriguez M, Barroso J (1987) Lateralization in male rats anddopaminergic system: evidence of right-side population bias. Physiol Behav 40:607-612

Chivers DP, McCormick MI, Allan Bridie JM, Mitchell MD, Gonçalves EJ, Bryshun R, Ferrari MCO (2016) At odds with the group: changes in lateralization and escape performance reveal conformity and conflict in fish schools. Proc Biol Sci. https://doi. org/10.1098/rspb.2016.1127

Cleal M, Parker MO (2018) Moderate developmental alcohol exposure reduces repetitive alternation in a zebrafish model of fetal alcohol spectrum disorders. Neurotoxicol Teratol 70:1-9. https://doi. org/10.1016/j.ntt.2018.09.001

Corballis MC (1986) Brain asymmetries: cerebral lateralization in nonhuman species. Science 231:1022-1023. https://doi.org/10.1126/ science.231.4741.1022-a

Dadda M, Domenichini A, Piffer L, Argenton F, Bisazza A (2010a) Early differences in epithalamic left-right asymmetry influence lateralization and personality of adult zebrafish. Behav Brain Res 206:208-215. https://doi.org/10.1016/j.bbr.2009.09.019 
Dadda M, Koolhaas WH, Domenici P (2010b) Behavioural asymmetry affects escape performance in a teleost fish. Biol Let 6:414-417. https://doi.org/10.1098/rsbl.2009.0904

de Perera TB, Braithwaite VA (2005) Laterality in a non-visual sensory modality - the lateral line of fish. Curr Biol CB 15:R241-242. https://doi.org/10.1016/j.cub.2005.03.035

Diaz Palarea MD, Gonzalez MC, Rodriguez M (1987) Behavioral lateralization in the T-maze and monoaminergic brain asymmetries. Physiol Behav 40:785-789

Egan RJ et al (2009) Understanding behavioral and physiological phenotypes of stress and anxiety in zebrafish. Behav Brain Res 205:38-44. https://doi.org/10.1016/j.bbr.2009.06.022

Fagot J, Vauclair J (1991) Manual laterality in nonhuman primates: a distinction between handedness and manual specialization. Psychol Bull 109:76-89

Fontana BD, Mezzomo NJ, Kalueff AV, Rosemberg DB (2018) The developing utility of zebrafish models of neurological and neuropsychiatric disorders: a critical review. Exp Neurol 299:157171. https://doi.org/10.1016/j.expneurol.2017.10.004

Franklin RG Jr, Adams RB Jr (2010) The two sides of beauty: laterality and the duality of facial attractiveness. Brain Cogn 72:300-305. https://doi.org/10.1016/j.bandc.2009.10.002

Frasnelli E (2013) Brain and behavioral lateralization in invertebrates. Front Psychol 4:939. https://doi.org/10.3389/fpsyg.2013.00939

Frasnelli E, Vallortigara G, Rogers LJ (2010) Response competition associated with right-left antennal asymmetries of new and old olfactory memory traces in honeybees. Behav Brain Res 209:3641. https://doi.org/10.1016/j.bbr.2010.01.014

Frasnelli E, Vallortigara G, Rogers LJ (2012) Left-right asymmetries of behaviour and nervous system in invertebrates. Neurosci Biobehav Rev 36:1273-1291. https://doi.org/10.1016/j.neubi orev.2012.02.006

Fride E, Weinstock M (1989) Alterations in behavioral and striatal dopamine asymmetries induced by prenatal stress. Pharmacol Biochem Behav 32:425-430

Ghafouri S, Fathollahi Y, Javan M, Shojaei A, Asgari A, MirnajafiZadeh J (2016) Effect of low frequency stimulation on impaired spontaneous alternation behavior of kindled rats in Y-maze test. Epilepsy Res 126:37-44. https://doi.org/10.1016/j.eplepsyres .2016 .06 .010

Gross AN, Engel AK, Richter SH, Garner JP, Wurbel H (2011) Cageinduced stereotypies in female ICR CD-1 mice do not correlate with recurrent perseveration. Behav Brain Res 216:613-620. https ://doi.org/10.1016/j.bbr.2010.09.003

Gunturkun O, Hellmann B, Melsbach G, Prior H (1998) Asymmetries of representation in the visual system of pigeons. NeuroReport 9:4127-4130

Hopkins WD (1994) Hand preferences for bimanual feeding in 140 captive chimpanzees (Pan troglodytes): rearing and ontogenetic determinants. Dev Psychobiol 27:395-407. https://doi.org/10.1002/ dev.420270607

Hull R, Vaid J (2007) Bilingual language lateralization: a meta-analytic tale of two hemispheres. Neuropsychologia 45:1987-2008. https ://doi.org/10.1016/j.neuropsychologia.2007.03.002

Hunt ER, O'Shea-Wheller T, Albery GF, Bridger TH, Gumn M, Franks NR (2014) Ants show a leftward turning bias when exploring unknown nest sites. Biol Lett 10:20140945. https://doi. org/10.1098/rsbl.2014.0945

Kalueff AV et al (2013) Towards a comprehensive catalog of zebrafish behavior 1.0 and beyond. Zebrafish 10:70-86. https://doi. org/10.1089/zeb.2012.0861

Kool W, McGuire JT, Rosen ZB, Botvinick MM (2010) Decision making and the avoidance of cognitive demand. J Exp Psychol Gen 139:665-682. https://doi.org/10.1037/a0020198

Koster EH, De Raedt R, Leyman L, De Lissnyder E (2010) Moodcongruent attention and memory bias in dysphoria: exploring the coherence among information-processing biases. Behav Res Ther 48:219-225. https://doi.org/10.1016/j.brat.2009.11.004

Lauzon NM, Bishop SF, Laviolette SR (2009) Dopamine D1 versus D4 receptors differentially modulate the encoding of salient versus nonsalient emotional information in the medial prefrontal cortex. J Neurosci Off J Soc Neurosci 29:4836-4845. https://doi. org/10.1523/jneurosci.0178-09.2009

Laviolette SR, Lipski WJ, Grace AA (2005) A subpopulation of neurons in the medial prefrontal cortex encodes emotional learning with burst and frequency codes through a dopamine D4 receptor-dependent basolateral amygdala input. J Neurosci Off J Soc Neurosci 25:6066-6075. https://doi.org/10.1523/jneur osci.1168-05.2005

Levin ED, Bencan Z, Cerutti DT (2007) Anxiolytic effects of nicotine in zebrafish. Physiol Behav 90:54-58. https://doi.org/10.1016/j. physbeh.2006.08.026

Lewis SA, Negelspach DC, Kaladchibachi S, Cowen SL, Fernandez F (2017) Spontaneous alternation: a potential gateway to spatial working memory in Drosophila. Neurobiol Learn Mem 142:230 235. https://doi.org/10.1016/j.nlm.2017.05.013

Lichtenstein-Vidne L, Okon-Singer H, Cohen N, Todder D, Aue T, Nemets B, Henik A (2017) Attentional bias in clinical depression and anxiety: the impact of emotional and non-emotional distracting information. Biol Psychol 122:4-12. https://doi.org/10.1016/j. biopsycho.2016.07.012

Lychakov DV (2013) Lateralization of behavioral reactions and otolith asymmetry. Zhurnal evoliutsionnoi biokhimii i fiziologii 49:299-312

Manuel R, Gorissen M, Roca CP, Zethof J, van de Vis H, Flik G, van den Bos R (2014) Inhibitory avoidance learning in zebrafish (Danio rerio): effects of shock intensity and unraveling differences in task performance. Zebrafish 11:341-352. https://doi. org/10.1089/zeb.2013.0970

Manuel R, Zethof J, Flik G, van den Bos R (2015) Providing a food reward reduces inhibitory avoidance learning in zebrafish. Behav Proc 120:69-72. https://doi.org/10.1016/j.beproc.2015.08.013

Maximino C, de Brito TM, da Silva Batista AW, Herculano AM, Morato S, Gouveia A Jr (2010) Measuring anxiety in zebrafish: a critical review. Behav Brain Res 214:157-171. https://doi. org/10.1016/j.bbr.2010.05.031

McGrew WC, Marchant LF (1997) On the other hand: current issues in and meta-analysis of the behavioral laterality of hand function in nonhuman primates. Phys Anthropol 104:201-232

Mezzomo NJ, Silveira A, Giuliani GS, Quadros VA, Rosemberg DB (2016) The role of taurine on anxiety-like behaviors in zebrafish: a comparative study using the novel tank and the light-dark tasks. Neurosci Lett 613:19-24. https://doi.org/10.1016/j.neule t.2015.12.037

Nakagawa M, Ohgoh M, Nishizawa Y, Ogura H (2004) Dopaminergic agonists and muscarinic antagonists improve lateralization in hemiparkinsonian rats in a novel exploratory Y-maze. J Pharmacol Exp Ther 309:737-744. https://doi.org/10.1124/jpet.103.059519

Nepomnyashchikh GI, Izvekov EI (2006) Laterality of behavioral responses in bony fishes: inheritance, adaptive importance, and morphofunctional correlates. J Ichthyol 47:782-790

Neveu PJ (1996) Lateralization and stress responses in mice: Interindividual differences in the association of brain, neuroendocrine, and immune responses. Behav Genet 26:373-377

Ng MC, Hsu CP, Wu YJ, Wu SY, Yang YL, Lu KT (2012) Effect of MK-801-induced impairment of inhibitory avoidance learning in zebrafish via inactivation of extracellular signal-regulated kinase (ERK) in telencephalon. Fish Physiol Biochem 38:1099-1106. https://doi.org/10.1007/s10695-011-9595-8

Ocklenburg S, Korte SM, Peterburs J, Wolf OT, Gunturkun O (2016) Stress and laterality - the comparative perspective. Physiol Behav 164:321-329. https://doi.org/10.1016/j.physbeh.2016.06.020 
Oliveira RF (2013) Mind the fish: zebrafish as a model in cognitive social neuroscience. Front Neural Circ 7:131. https://doi. org/10.3389/fncir.2013.00131

Parker MO, Millington ME, Combe FJ, Brennan CH (2012) Housing conditions differentially affect physiological and behavioural stress responses of zebrafish, as well as the response to anxiolytics. PloS One 7:e34992. https://doi.org/10.1371/journ al.pone.0034992

Pickering C, Alsio J, Morud J, Ericson M, Robbins TW, Soderpalm B (2015) Ethanol impairment of spontaneous alternation behaviour and associated changes in medial prefrontal glutamatergic gene expression precede putative markers of dependence. Pharmacol Biochem Behav 132:63-70. https://doi.org/10.1016/j. pbb.2015.02.021

Pisa M, Szechtman H (1986) Lateralized and compulsive exteroceptive orientation in rats treated with apomorphine. Neurosci Lett 64:41-46

Reale D, Reader SM, Sol D, McDougall PT, Dingemanse NJ (2007) Integrating animal temperament within ecology and evolution. Biol Rev Camb Philos Soc 82:291-318. https://doi.org/10.1111/ j.1469-185X.2007.00010.x

Reddon AR, Balshine S (2010) Lateralization in response to social stimuli in a cooperatively breeding cichlid fish. Behav Proc 85:6871. https://doi.org/10.1016/j.beproc.2010.06.008

Ridley RM (1994) The psychology of perserverative and stereotyped behaviour. Prog Neurobiol 44:221-231

Rigosi E, Frasnelli E, Vinegoni C, Antolini R, Anfora G, Vallortigara G, Haase A (2011) Searching for anatomical correlates of olfactory lateralization in the honeybee antennal lobes: a morphological and behavioural study. Behav Brain Res 221:290-294. https ://doi.org/10.1016/j.bbr.2011.03.015

Robison DN (1981) Conceptual aspects of "laterality" syndromes. Behav Brain Sci 4:33-34

Rodriguez M, Afonso D (1993) Ontogeny of T-maze behavioral lateralization in rats. Physiol Behav 54:91-94

Rodriguez M, Gomez C, Alonso J, Afonso D (1992) Laterality, alternation, and perseveration relationships on the T-maze test. Behav Neurosci 106:974-980

Rogers LJ (2000) Evolution of hemispheric specialization: advantages and disadvantages. Brain Lang 73:236-253. https://doi. org/10.1006/brln.2000.2305

Rogers LJ (2002) Lateralization in vertebrates: its early evolution, general pattern and development. Adv Study Behav Brain Sci 31:107-162

Rogers LJ, Vallortigara G (2008) From antenna to antenna: lateral shift of olfactory memory recall by honeybees. PloS One 3:e2340. https ://doi.org/10.1371/journal.pone.0002340
Rosemberg DB et al (2012) Behavioral effects of taurine pretreatment in zebrafish acutely exposed to ethanol. Neuropharmacology 63:613-623. https://doi.org/10.1016/j.neuropharm.2012.05.009

Sovrano VA, Andrew RJ (2006) Eye use during viewing a reflection: behavioural lateralisation in zebrafish larvae. Behav Brain Res 167:226-231. https://doi.org/10.1016/j.bbr.2005.09.021

Sovrano VA, Dadda M, Bisazza A (2005) Lateralized fish perform better than non-lateralized fish in spatial reorientation tasks. Behav Brain Res 163:122-127. https://doi.org/10.1016/j.bbr.2005.04.012

Stewart AM, Ullmann JF, Norton WH, Parker MO, Brennan CH, Gerlai R, Kalueff AV (2015) Molecular psychiatry of zebrafish. Mol Psychiatry 20:2-17. https://doi.org/10.1038/mp.2014.128

Tran S, Nowicki M, Muraleetharan A, Chatterjee D, Gerlai R (2016) Neurochemical factors underlying individual differences in locomotor activity and anxiety-like behavioral responses in zebrafish. Prog Neuropsychopharmacol Biol Psychiatry 65:25-33. https:// doi.org/10.1016/j.pnpbp.2015.08.009

Valente A, Huang KH, Portugues R, Engert F (2012) Ontogeny of classical and operant learning behaviors in zebrafish. Learn Mem 19:170-177. https://doi.org/10.1101/lm.025668.112

Vallortigara G, Rogers LJ (2005) Survival with an asymmetrical brain: advantages and disadvantages of cerebral lateralization. Behav Brain Sci 28:575-589. https://doi.org/10.1017/s0140525x050001 05

Vikingstad EM, George KP, Johnson AF, Cao Y (2000) Cortical language lateralization in right handed normal subjects using functional magnetic resonance imaging. J Neurol Sci 175:17-27

Wahlsten D (1990) Insensitivity of the analysis of variance to heredityenvironment interaction. Behav Brain Sci 13:109-120

Westergaard GC, Lussier ID, Suomi SJ, Higley JD (2001) Stress correlates of hand preference in rhesus macaques. Dev Psychobiol $38: 110-115$

Wong $\mathrm{K}$ et al (2010) Analyzing habituation responses to novelty in zebrafish (Danio rerio). Behav Brain Res 208:450-457. https:// doi.org/10.1016/j.bbr.2009.12.023

Zimmerberg B, Glick SD (1974) Rotation and stereotypy during electrical stimulation of the caudate nucleus. Res Commun Chem Pathol Pharmacol 8:195

Publisher's Note Springer Nature remains neutral with regard to jurisdictional claims in published maps and institutional affiliations. 